\title{
1 A comparison of methodological approaches to the study of young sex chromosomes: A case study in
}

\section{Poecilia}

3 Iulia Darolti ${ }^{1}$, Pedro Almeida ${ }^{2}$, Alison E. Wright ${ }^{3}$, Judith E. Mank ${ }^{1,4}$

41 Department of Zoology and Biodiversity Research Centre, University of British Columbia, Vancouver,

5 Canada

62 Department of Genetics, Evolution and Environment, University College London, London, United

7 Kingdom

83 Ecology and Evolutionary Biology, School of Biosciences, University of Sheffield, Sheffield, United

9 Kingdom

104 Centre for Ecology and Conservation, College of Life and Environmental Sciences, University of Exeter,

11 Cornwall, United Kingdom

12 Co-corresponding author: Judith E. Mank, e-mail: mank@zoology.ubc.ca

13 Co-corresponding author: Iulia Darolti, e-mail: darolti@zoology.ubc.ca 


\section{Abstract}

15 Studies of sex chromosome systems at early stages of divergence are key to understanding the initial 16 process and underlying causes of recombination suppression. However, identifying signatures of

17 divergence in homomorphic sex chromosomes can be challenging due to high levels of sequence similarity

18 between the $\mathrm{X}$ and the $\mathrm{Y}$. Variations in methodological precision and underlying data can make all the

19 difference between detecting subtle divergence patterns or missing them entirely. Recent efforts to test

20 for $X-Y$ sequence differentiation in the guppy have led to contradictory results. Here we apply different

21 analytical methodologies to the same dataset to test for the accuracy of different approaches in

22 identifying patterns of sex chromosome divergence in the guppy. Our comparative analysis reveals that

23 the most substantial source of variation in the results of the different analyses lies in the reference

24 genome used. Analyses using custom-made de novo genome assemblies for the focal species successfully

25 recover a signal of divergence across different methodological approaches. By contrast, using the distantly

26 related Xiphophorus reference genome results in variable patterns, due to both sequence evolution and

27 structural variations on the sex chromosomes between the guppy and Xiphophorus. Changes in mapping

28 and filtering parameters can additionally introduce noise and obscure the signal. Our results illustrate how

29 analytical differences can alter perceived results and we highlight best practices for the study of nascent

30 sex chromosomes. 


\section{Introduction}

32 Substantial recent attention in sex chromosome research has focused on the earliest stages of $X-Y$ divergence in order to glean the initial processes of recombination suppression (Wright et al. 2016). Studies of nascent sex chromosome divergence will by definition result in subtle patterns of $X-Y$ sequence differentiation as substantial differences have not yet sufficiently accumulated. Given the expected subtlety, methodology and underlying data can be quite important, and small changes may make all the difference between identifying a delicate pattern or missing it entirely.

For example, several recent tests for divergence between the guppy $\mathrm{X}$ and $\mathrm{Y}$ chromosomes have revealed contradictory results. Full genomic analysis of the Poecilia reticulata sex chromosomes was originally presented in Wright et al. (2017) based on comparisons between male and female genomes (Fig. 1). This approach can be used to identify what, if any, regions of the $Y$ chromosome are diverged from the $X$, and to compare across populations to determine intra-specific variation. Wright et al. (2017) found a relatively small region $(10 \mathrm{Mb})$ of significant $Y$ degeneration, designated Stratum I. This region was characterized by a reduction in the number of male reads that mapped compared to females, consistent with the concept of $\mathrm{Y}$ degeneration. Moreover, the same pattern was observed in all six of the natural populations assayed as well as a captive lab population, and the rules of parsimony therefore suggest that Stratum I is ancestral to the colonization of Trinidad. Wright et al. (2017) also observed evidence of a second region of nascent divergence, Stratum II, that appeared to have formed independently in three upstream populations, but was smaller in downstream populations. This region was characterized by an increase in male single nucleotide polymorphism (SNP) density compared to females but no degradation of the $\mathrm{Y}$. This pattern is consistent with either greatly reduced or complete loss of male recombination in this region, or selection against recombinant males.

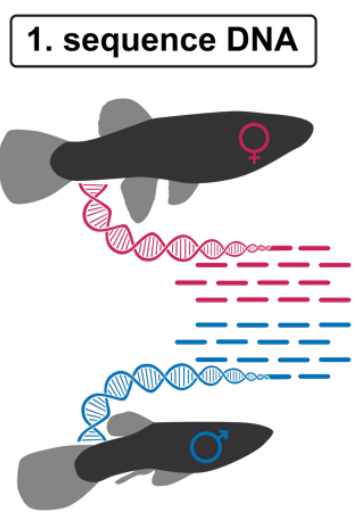

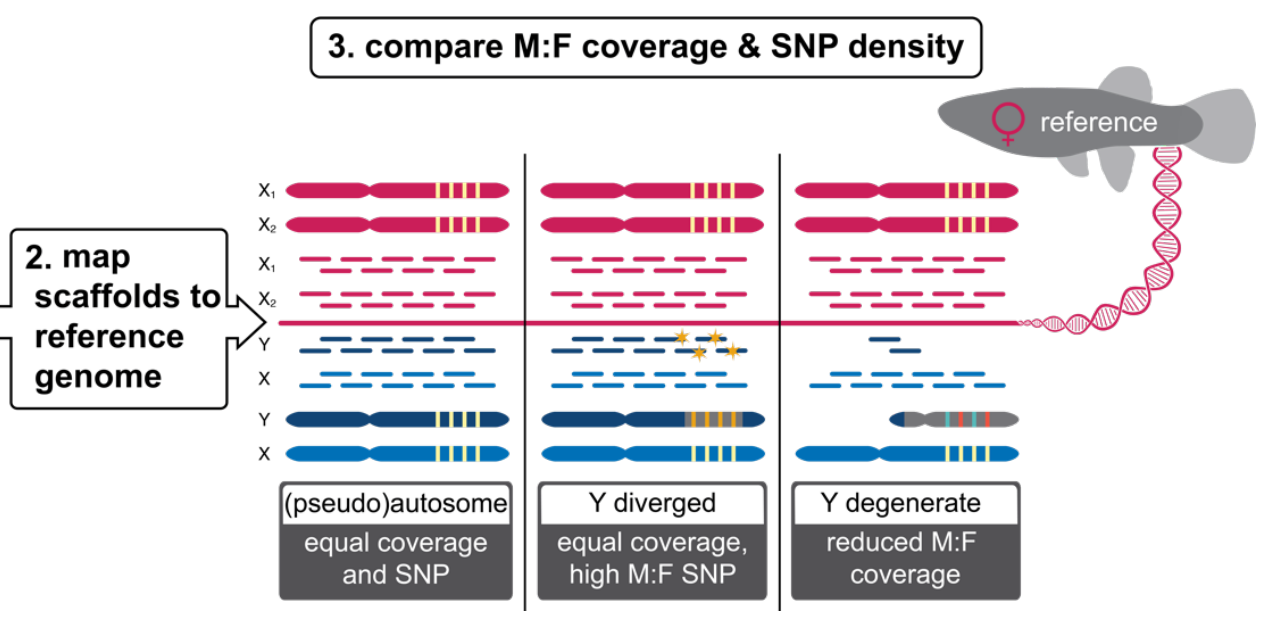


Figure 1. Genomic comparisons of male and female DNA data can be used to identify X-Y divergence. Step (1) Multiple males and females are sequenced, and female reads (red) are assembled, with resulting scaffolds ordered and oriented to the nearest available full reference genome. Step (2) Male (blue) and female (red) reads are mapped to this assembly. Step (3) $Y$ divergence leads to male-specific SNPs, and therefore elevated male:female SNP density. As the $Y$ degenerates, $Y$ reads will no longer map to the $X$ chromosome assembly, leading to reduced male:female coverage. Method adapted from Vicoso \& Bachtrog 2013 and Vicoso and Bachtrog 2015. Figure courtesy of Jacelyn Shu (jacelyndesigns.com).

Almeida et al. (2021) built on these initial findings with a greatly expanded dataset, again recovering concordant patterns of Stratum I across the same six natural populations of $P$. reticulata. The expanded dataset incorporated 10X genomics linked-reads, allowing for far more sophisticated analyses. Namely by phasing $\mathrm{X}$ and $\mathrm{Y}$ haplotypes, it was possible to discern that Stratum I is comprised of two smaller separate regions of reduced male:female read depth. This region is also enriched for male-specific sequences, malespecific SNPs, and repetitive elements, the presence of which necessitate recombination suppression from the $X$ chromosome. Importantly, there was also evidence of phylogenetic clustering of phased $Y$ sequence in this region, indicating ancestral recombination suppression. Finally, this replication recovered evidence of parallel expansion of Stratum II in upstream populations.

Expanding phylogenetically, Darolti et al (2019) uncovered consistent patterns of sex chromosome topology in $P$. wingei. Initial karyotype analysis suggested that the $\mathrm{X}$ and $\mathrm{Y}$ chromosomes are ancestral to the common guppy (P. reticulata) and Endler's Guppy (P. wingei) (Nanda et al. 1993). Furthermore, Darolti et al. (2019) found the same small region of Y chromosome degeneration consistent with Stratum I, although somewhat more pronounced in the degree of divergence from the $\mathrm{X}$ than $P$. reticulata. The region of degeneration matched nearly perfectly with $P$. reticulata, suggesting Stratum I was in fact present in the common ancestor of $P$. wingei and $P$. reticulata. Consistent with this, Morris et al (2018) found evidence of male-specific sequence shared between $P$. reticulata and $P$. wingei, possible only if recombination between the $X$ and $Y$ was halted in the common ancestor of these species. Moreover, Darolti et al. (2020) used SNP segregation patterns from RNA-seq data across pedigrees to determine $X$ and $Y$ sequence, and found four genes that showed phylogenetic evidence of recombination suppression in the ancestor of $P$. wingei and $P$. reticulata. Although the bootstrap values for any one locus were not excessively high, it is telling that all four were in Stratum I. The ancestral origin of Stratum I was further supported by conserved patterns of male-hypomethylation within this region in both species (Metzger et al. 2020), consistent with sexualization of gene regulation. Finally, Darolti et al. (2019) found evidence for another independent origin of Stratum II based on SNP data in P. wingei. Work in outgroup species revealed the same chromosome is a sex chromosome in P. picta and P. parae (Darolti et al. 2019; Sandkam et al. 2021), although diverged to a far greater degree in both these species. 
Crucially, all of these analyses were based on custom genome or transcriptome assemblies generated bespoke from the underlying data (Wright et al. 2017; Darolti et al. 2019; Darolti et al. 2020; Almeida et al. 2021), although they did use existing related reference genomes to physically place and orient scaffolds. This is in contrast to other studies which have used existing resources derived from different populations or species, resulting in potential mismatches between the underlying data and the genome to which it is compared. Taking a bespoke approach is critical as it reduces the phylogenetic distance

94 between the sequence reads and the reference to which they are mapped, which can increase the proportion of reads that are accurately mapped and reduce issues arising from structural variation and repetitive sequence. Secondly, an important step in identifying diverged regions in sex chromosomes is ensuring stringent mapping parameters (Caravalho and Clark 2013; Smeds et al. 2015; Vicoso and Bachtrog 2013; Vicoso and Bachtrog 2015; Palmer et al. 2019). This is particularly relevant for homomorphic sex chromosomes as they still retain sequence orthology between the $\mathrm{X}$ and $\mathrm{Y}$, and incorrectly mapped reads can mask coverage differences between the sexes and lead to the misclassification of sex-linked sequences as autosomal. Wright et al. (2017), Darolti et al. (2019) and Almeida et al. (2021) used stringent mapping limits, removed minor alleles with low frequency, which

103 likely represent sequencing errors, and focused on coding sequence to minimize issues with repetitive 104 elements (Table 1). This was based on the reasoning that young sex chromosomes would exhibit subtle 105 divergence signatures, and stringency would be required to detect it (Palmer et al. 2019; Vicoso and 106 Bachtrog 2015). 
Table 1. A comparison of methods and findings for $P$. reticulata sex chromosome strata

\begin{tabular}{|c|c|c|c|c|c|c|}
\hline Study & $\begin{array}{l}\text { Evidence for } \\
\text { Stratum I } \\
\text { from } M: F \\
\text { read depth }\end{array}$ & $\begin{array}{l}\text { Phylogenetic } \\
\text { clustering of } Y \\
\text { sequences in } \\
\text { Stratum I }\end{array}$ & $\begin{array}{l}\text { Evidence for } \\
\text { Stratum II } \\
\text { from M:F FsT }\end{array}$ & $\begin{array}{l}\text { Genome } \\
\text { Assembly }\end{array}$ & $\begin{array}{l}\text { Read depth } \\
\text { analysis }\end{array}$ & SNP analysis \\
\hline $\begin{array}{l}\text { Wright et al. } \\
\text { (2017) }\end{array}$ & Yes & $\mathrm{n} / \mathrm{a}$ & Yes & $\begin{array}{c}\text { Bespoke } \\
\text { (P. reticulata) }\end{array}$ & $\begin{array}{l}\text { Uniquely mapping } \\
\text { reads }\end{array}$ & $\begin{array}{l}\text { Limited to coding } \\
\text { sequence; Site } \\
\text { coverage }>10 ; \text { SNP } \\
\text { frequency }>0.3 x \\
\text { site coverage }\end{array}$ \\
\hline $\begin{array}{l}\text { Bergero et al. } \\
\text { (2019) }\end{array}$ & No & $\mathrm{n} / \mathrm{a}$ & Yes & $\begin{array}{l}\text { Kunstner et al. } \\
\qquad(2016) \\
\text { (P. reticulata) }\end{array}$ & $\begin{array}{l}\text { Default mismatch } \\
\text { parameters, } \\
\text { duplicate reads } \\
\text { excluded }\end{array}$ & $\begin{array}{l}\text { Quality score > 30; } \\
\text { Minimum coverage } \\
\text { 20; Biallelic SNPs }\end{array}$ \\
\hline $\begin{array}{l}\text { Darolti et al. } \\
\quad(2019)\end{array}$ & Yes & $\mathrm{n} / \mathrm{a}$ & Yes & $\begin{array}{c}\text { Bespoke } \\
\text { (P. reticulata } \\
\text { and } P \text {. wingei) }\end{array}$ & $\begin{array}{l}\text { Uniquely mapping } \\
\text { read }\end{array}$ & $\begin{array}{l}\text { Limited to coding } \\
\text { sequence; Site } \\
\text { coverage }>10 ; \text { SNP } \\
\text { frequency }>0.3 x \\
\text { site coverage }\end{array}$ \\
\hline $\begin{array}{l}\text { Charlesworth } \\
\text { et al. (2020) }\end{array}$ & No & $\mathrm{n} / \mathrm{a}$ & Yes & $\begin{array}{l}\text { Kunstner et al. } \\
\qquad(2016) \\
\text { (P. reticulata) }\end{array}$ & Not reported* & Not reported* \\
\hline $\begin{array}{l}\text { Fraser et al. } \\
\quad(2020)\end{array}$ & Yes & $\mathrm{n} / \mathrm{a}$ & No & $\begin{array}{c}\text { Bespoke } \\
\text { (P. reticulata) }\end{array}$ & $\begin{array}{l}\text { Default mismatch } \\
\text { parameters }\end{array}$ & MAF $>0.05$ \\
\hline $\begin{array}{l}\text { Kirkpatrick et } \\
\text { al. (2020) }\end{array}$ & No & Yes & No & $\begin{array}{l}\text { Schartl et al. } \\
\text { (2013) } \\
\text { (Xiphophorus } \\
\text { maculatus) }\end{array}$ & $\begin{array}{l}\text { Default read } \\
\text { mapping } \\
\text { parameters with } \\
\text { local argument, } \\
\text { duplicate reads } \\
\text { excluded }\end{array}$ & $\begin{array}{c}\text { Quality score > 20; } \\
\text { Minimum read } \\
\text { depth 3; Biallelic } \\
\text { SNPs }\end{array}$ \\
\hline $\begin{array}{l}\text { Almeida et al. } \\
\qquad(2021)\end{array}$ & Yes & Yes & Yes & $\begin{array}{c}\text { Bespoke } \\
\text { (P. reticulata, } \\
\text { river-specific) }\end{array}$ & $\begin{array}{l}\text { Uniquely mapping } \\
\text { reads, duplicate } \\
\text { reads excluded }\end{array}$ & $\begin{array}{c}\text { MAF > 0.1; } \\
\text { excluding } \\
\text { extremely high } \\
\text { coverage sites }\end{array}$ \\
\hline
\end{tabular}

*Mapping criteria not reported in methods and bioinformatic code not publicly available. Defaults assumed. MAF = minor allele frequency.

109 Despite observing remarkable concordance of these patterns across multiple datasets, species and 110 analytical methods, other recent studies have differed substantially in their approach and reported some 111 different results (Table 1). For example, Bergero et al. (2019) did not report evidence for Stratum I in their 112 own P. reticulata data, and although they did uncover a pattern that is broadly consistent with Stratum II, 113 it was not statistically different across populations (Charlesworth et al. 2020). Fraser et al. (2020) 114 identified small male-specific regions largely consistent with the regions identified by Almeida et al. 115 (2021), although because of scaffold orientation differences and population-specific inversions, they are 
in different physical locations. Finally, Kirkpatrick et al. (2020), reanalyzing data from Darolti et al. (2019) found evidence of Stratum I and Stratum II in P. wingei, but not in P. reticulata. Notably, they did find phylogenetic evidence of recombination suppression in the ancestor of these two species in Stratum I, consistent with Darolti et al. (2020) and Almeida et al. (2021).

Importantly Bergero et al. (2019), Charlesworth et al. (2020) and Kirkpatrick et al. (2020) relied on existing reference genomes for all their analyses and did not use genomic reads to build custom-made assemblies for the target species. Importantly, Kirkpatrick et al. (2020) mapped reads from $P$. wingei and $P$. reticulata to Xiphophorus, which last shared a common ancestor 40 mya (Kumar et al. 2017). Additionally, Bergero et al. (2019) used less stringent mapping criteria, and Charlesworth et al. (2020) and Fraser et al. (2020) used default mapping parameters. Together, this produces two sources of potential methodological noise in replication efforts. First, noise can arise from accumulated mutations due to phylogenetic distance between the samples used to generate sequence reads and the genome that they are mapped to. Second, permissive default mapping parameters allow for mismapping, and therefore potentially result in significant noise in genomic comparisons between males and females. In addition to this, many of these studies used different underlying datasets that varied in sample origin, number and read depth, and so it is difficult to distinguish the role of sample variation from methodological differences in these discrepancies.

The proliferation of studies on this system with different levels of analytical sophistication allow for a remarkable comparison of the role of genomic methodology in pattern discovery. We tested various methods on the same underlying data with the goal of determining the methodological reasons for inconsistent findings across these studies, and to develop best practices moving forward to the genomic study of nascent sex chromosome systems.

\section{Methods}

\section{Datasets}

Using the $P$. reticulata data from Almeida et al. (2021) and the $P$. wingei data from Darolti et al. (2019), we ran multiple analyses of guppy sex chromosome evolution, following the various analytical methods used by Wright et al. (2017), Bergero et al. (2019) and Kirkpatrick et al. (2020), as summarized in Table 1 and detailed below. We were unable to include the methodology of Charlesworth et al. (2020) as mapping criteria was not reported in methods and bioinformatic code is not publicly available. The datasets for $P$. reticulata and $P$. wingei included paired-end DNA-seq reads from three males and three females from the 
Quare upstream population (EBI ENA under BioProject PRJEB39998) and from our lab population (NCBI SRA under BioProject PRJNA528814), respectively. We assessed read quality using FastQC v0.11.9 (www.bioinformatics.babraham.ac.uk/projects/fastqc/, last accessed 8 November 2021), trimmed using Trimmomatic v0.36 (Bolger et al. 2014) and concatenated reads as in Darolti et al. (2019) and Almeida et al. (2021). To replicate previous studies, all analyses were repeated using several different genomes and their respective gene annotations, which included the $P$. reticulata Quare de novo genome assembly from Almeida et al. (2021), the P. reticulata reference genome from Kunstner et al. (2016) (NCBI accession GCF_000633615.1), the P. wingei de novo genome assembly from Darolti et al. (2019) and the Xiphophorus maculatus reference genome from Schartl et al. (2013) (NCBI accession GCF_002775205.2, v5.0).

\section{Coverage analysis}

156 For each focal species, we used three separate methodological pipelines to map and filter reads and to estimate read depth. The first method followed the analysis in Wright et al. (2017), which used bwa v0.7.15 aln/sampe (Li and Durbin 2009) to map reads, removed reads that were not uniquely mapping and estimated coverage with soap.coverage v2.7.7 (http://soap.genomics.org.cn, last accessed 1 April 2019). The second method followed the pipeline in Kirkpatrick et al. (2020), which mapped reads using bowtie2 v2.2.9 with default parameters and the -local argument (Langmead and Salzberg 2012), removed PCR duplicates using Picard v2.0.1 (http://broadinstitute.github.io/picard, last accessed 8 November 2021) and calculated coverage with BEDtools v2.26 (Quinlan and Hall 2010). Lastly, the third method followed the analysis in Bergero et al. (2019), which mapped reads with bwa mem and the -M argument

165 (Li and Durbin 2009), removed PCR duplicates with BEDtools (Quinlan and Hall 2010) and estimated coverage using SAMtools v1.3.1 (Li et al. 2009).

167 For all three methodological pipelines, average coverage values were calculated separately for males and

168 females, and average male:female coverage for each non-overlapping window was calculated as $169 \log _{2}$ (average male coverage) - $\log _{2}$ (average female coverage). A window size of $50 \mathrm{~kb}$ was used for all $P$. 170 reticulata analyses and $P$. wingei analyses based on the $X$. maculatus genome, while $10 \mathrm{~kb}$ windows were 171 used for $P$. wingei analyses using the more fragmented de novo $P$. wingei genome. Moving averages of 172 coverage were plotted in R v4.0.5 (R Core Team 2019) based on sliding window analyses using the 173 roll_mean function. Ninety-five percent confidence intervals for the moving average plots were obtained 174 by randomly sampling autosomal values 1,000 times without replacement. 
To further assess patterns of $Y$ divergence, for both $P$. reticulata and $P$. wingei, we compared three methodological approaches of estimating SNP density differences between males and females.

First, based on Wright et al. (2017), we mapped reads to each genome using bowtie2 with default parameters (Langmead and Salzberg 2012). After file sorting, we used bow2pro v0.1 (http://guanine.evolbio.mpg.de, last accessed 8 November 2021) to generate a profile for each sample, representing counts for each of the four nucleotide bases at each site. We then applied a minimum site coverage threshold of 10 and kept SNPs with a frequency of 0.3 times the site coverage. We further used gene annotation information to remove SNPs from the analysis if they were not located within coding sequences. For each sample, we calculated average SNP density for each gene as the sum of all SNPS divided by the sum of filtered sites in that gene, excluding those with zero filtered sites.

Second, following Kirkpatrick et al. (2020), we called variants from files previously filtered for PCR duplicates (see Coverage analysis section above) using BCFtools v.1.3.1 (Li 2011). We then filtered variants using VCFtools v0.1.12b (Danecek et al. 2011), removing indels and variants with a quality score lower than 20, and selecting for biallelic SNPs and a minimum read depth of 3. For each sample, we then used BEDtools counts to count the number of SNPs within 50kb windows across the genome.

Third, we used the pipeline in Bergero et al. (2019) to call SNPs from the PCR duplicates filtered files (see Coverage analysis section above) using GATK HaplotypeCaller v4.1.9 (Poplin et al. 2017) with the parameters --emit-ref-confidence GVCF and -stand-call-conf 30. Further genotyping was done with GATK GenotypeGVCFs with default parameters and SelectVariants to keep SNPs with a minimum coverage of 20, minimum quality of 30 and selecting for biallelic SNPs only. For each sample, we then used BEDtools counts to count the number of SNPs within $50 \mathrm{~kb}$ windows across the genome.

Lastly, in each of these three methodological approaches, average SNP density across all males and across all females was calculated separately. For each gene or window, we calculated male:female SNP density as $\log _{2}$ (average male SNP density) - $\log _{2}$ (average female SNP density). We then divided male:female SNP density estimates into autosomal and sex-linked based on chromosomal position. The distributions of male:female SNP density for the autosomes and the sex chromosomes were plotted in R ( $R$ Core Team 2019) and differences between them were tested using Wilcoxon rank sum tests.

Pairwise synteny analyses 
We used LAST v1256 (Kielbasa et al. 2011) to perform pairwise synteny analyses between the $P$. reticulata sex chromosome (chromosome 12) from the reference genome (Kunstner et al. 2016), the P. reticulata sex chromosome from the Quare de novo assembly (Almeida et al. 2021) and the X. maculatus syntenic chromosome 8. For alignments involving the $X$. maculatus sequence, we used LAST with the HOXD70 seeding scheme designed for a higher rate of substitution, whereas for alignments involving $P$. reticulata sequences only we used the UNEAR seeding scheme for aligning sequences with lower rate of substitutions.

\section{Results}

212 Using the same dataset across different genomes and methods, we first assessed the role of various 213 genomic analysis parameters (Table 1 ) in detecting Stratum I on the $P$. reticulata and $P$. wingei sex 214 chromosomes, previously reported in Wright et al. (2017), Darolti et al. (2019) and Almeida et al. (2021), 215 summarized in Fig. 2 and Fig. 3.

Analyses on $P$. reticulata sequencing data that used the custom-made de novo $P$. reticulata genome assembly show a significantly lower male to female coverage, indicative of $\mathrm{X}-\mathrm{Y}$ degeneration, at the distal end of the chromosome, in the previously estimated location of Stratum I (Fig. 2A, B). This pattern is evident from both the analysis that followed the pipeline from Wright et al. (2017) (Fig. 2A) and the analysis based on the methodology in Kirkpatrick et al. (2020) (Fig. 2B). All three analyses that relied on the $X$. maculatus reference genome also show a region with decreased male coverage relative to that in females, however, this region is shifted closer to the end of the chromosome and only partially overlaps with the syntenic region of the estimated location of $P$. reticulata Stratum I (Fig. 2D, E, F). Pairwise alignments revealed several structural rearrangements between the $P$. reticulata sex chromosome (chromosome 12) and the syntenic X. maculatus chromosome 8, particularly in the region of the predicted guppy Stratum I (Fig. 4), which may explain the shifted position of the region with low male coverage in analyses that use the $X$. maculatus genome. In addition, different methodological parameters can have a

228 significant impact on the proportion of reads mapped. Mapping efficiency is substantially reduced when using the $X$. maculatus reference (Table 2), which decreases power to detect a signal of X-Y differentiation.

We find no clear pattern of Stratum I when mapping reads to the $P$. reticulata reference genome based on the methodology in Bergero et al. (2019) (Fig. 2C). While we cannot disentangle between the reference genome used and the methodology in this analysis, our other data suggests that the absence of a Stratum 
to the $X$. maculatus genome we recover qualitatively the same coverage pattern across all three methodological approaches (Fig. 2D, E, F). Similarly, our P. wingei analyses detailed below reveal that, when using the same genome, the Bergero et al. (2019) pipeline produces very similar patterns to the assembly errors on the sex chromosome of the first draft of the $P$. reticulata reference genome (Bergero signal of Stratum I.

\section{Method}

Mapping: bwa -aln, -sampe Filtering: uniquely mapping reads Coverage: soap.coverage Wright et al. 2017

Mapping: bowtie2 -local Filtering: remove PCR duplicates Coverage: bedtools Kirkpatrick et al. 2020

Mapping: bwa -mem -M Filtering: remove PCR duplicates Coverage: samtools Bergero et al. 2019
P. reticulata

A

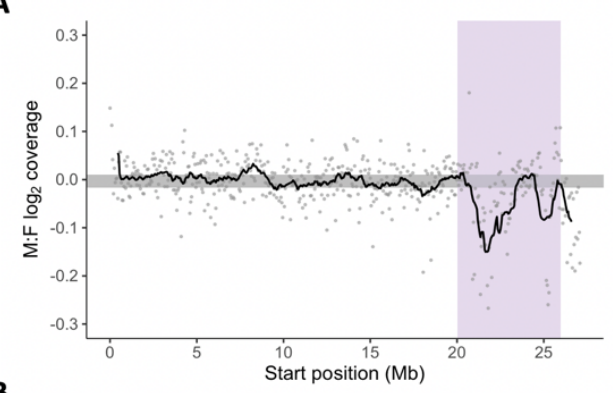

B

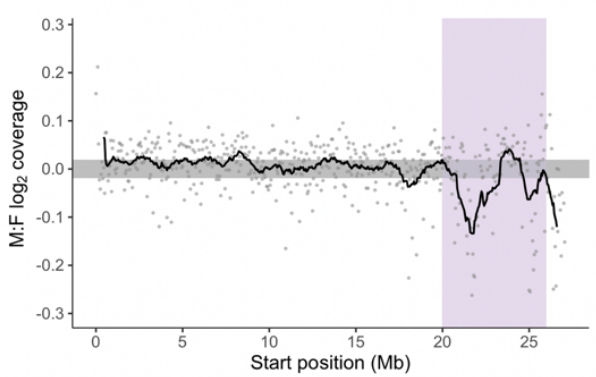

C

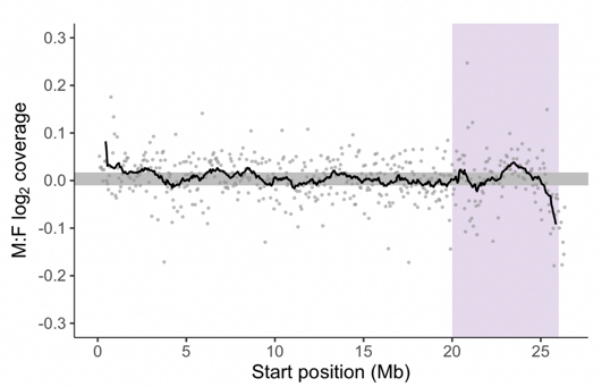

X. maculatus

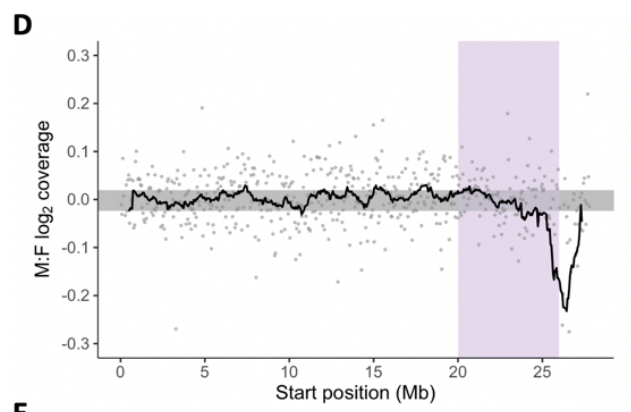

E

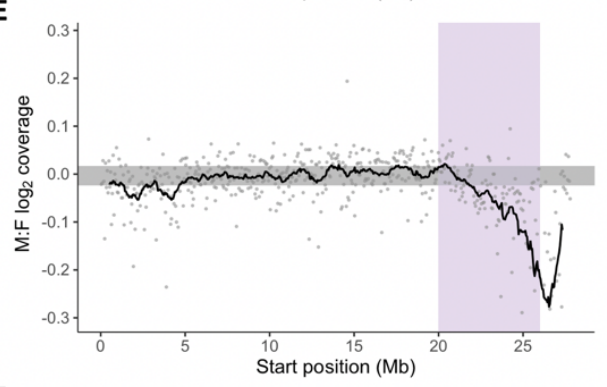

F

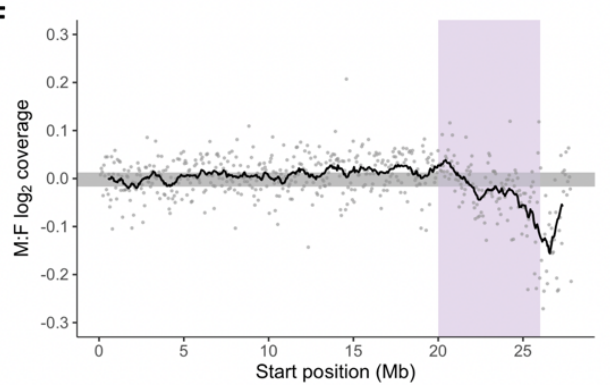

Figure 2. Signal for $P$. reticulata Stratum I using comparative methodological approaches. $P$. reticulata DNA-seq reads were mapped in turn to a $P$. reticulata genome assembly and the $X$. maculatus reference genome assembly (Schartl et al. 2013). For replicating previous studies, the $P$. reticulata reference genome from Kunstner et al. (2016) was used in the analysis based on the methods from Bergero et al. (2019), while the high quality Quare de novo assembly from Almeida et al. (2021) was used in the other two analyses. Moving average plots represent male to female coverage differences across the guppy sex chromosome ( $P$. reticulata chromosome 12 , and syntenic $X$. maculatus chromosome 8 ) in non-overlapping windows of $50 \mathrm{~kb}$. $95 \%$ confidence intervals, based on bootstrapping autosomal values, are shown in grey, and predicted boundaries for Stratum I from Almeida et al. (2021) are highlighted in purple. 
The analyses for $P$. wingei also reveal a lower male to female coverage at the distal end of the chromosome, however, this pattern is only observed in analyses that mapped reads to the de novo $P$. wingei assembly (Fig. 3A, B, C). By contrast, the analyses that used the $X$. maculatus genome all show a significantly elevated read depth in males compared to females, similar to the results in Kirkpatrick et al. (2020) (Fig. 3D, E, F). Previous cytogenetic work has shown that the $P$. wingei $Y$ chromosome is the largest chromosome in the genome, having accumulated a large heterochromatin block (Nanda et al. 2014). However, in addition to the expansion of repetitive sequence, duplication events from the rest of the genome could have also contributed to the remarkable size of the $P$. wingei $Y$ chromosome. Duplications relative to females in this species.

\section{Method}

Mapping: bwa -aln, -sampe Filtering: uniquely mapping reads Coverage: soap.coverage Wright et al. 2017

Mapping: bowtie2 -local Filtering: remove PCR duplicates Coverage: bedtools Kirkpatrick et al. 2020

Mapping: bwa -mem -M Filtering: remove PCR duplicates Coverage: samtools Bergero et al. 2019

\section{P. wingei}

A

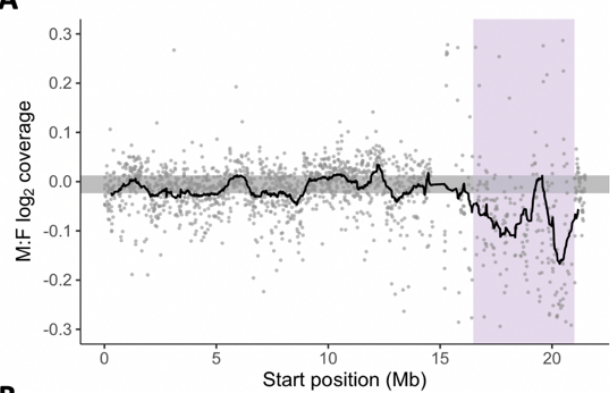

B

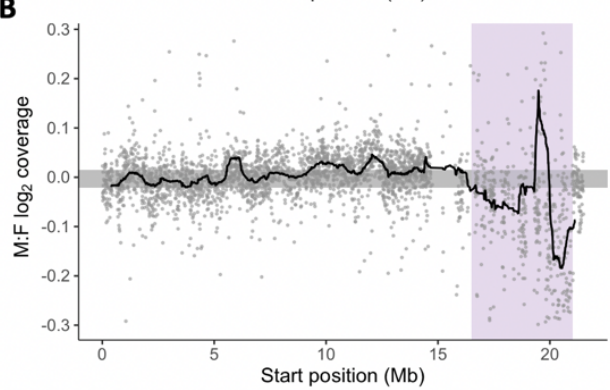

C

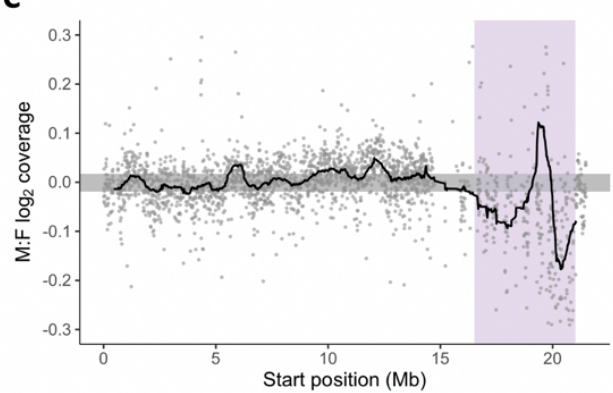

X. maculatus

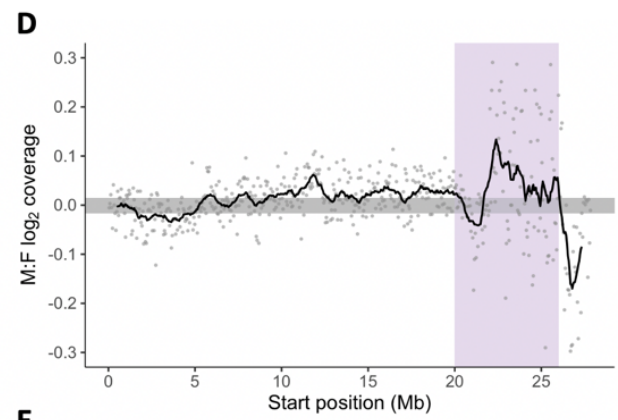

E

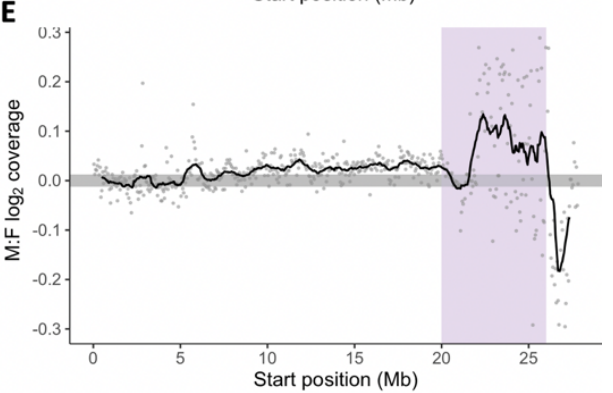

$\mathbf{F}$

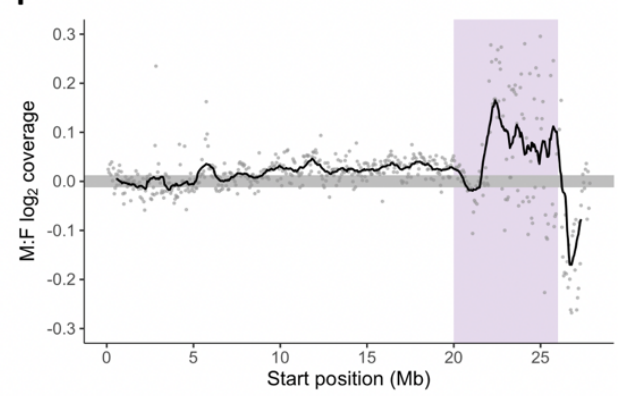

Figure 3. Signal for $\boldsymbol{P}$. wingei Stratum I using comparative methodological approaches. $P$. wingei DNA-seq reads were mapped in turn to a de novo $P$. wingei genome assembly (Darolti et al. 2019) and the $X$. maculatus reference genome assembly (Schartl et al. 2013). Moving average plots represent male to female coverage differences across the sex chromosome ( $P$. wingei chromosome 12 , and syntenic $X$. maculatus chromosome 8 ) in non-overlapping 
windows of $50 \mathrm{~kb}$ for the analyses that rely on the $X$. maculatus genome and windows of $10 \mathrm{~kb}$ for the analyses that use the de novo $P$. wingei genome. The $95 \%$ confidence intervals, based on bootstrapping autosomal values, are shown in grey, and predicted boundaries for Stratum I from Darolti et al. (2019) are highlighted in purple.

A

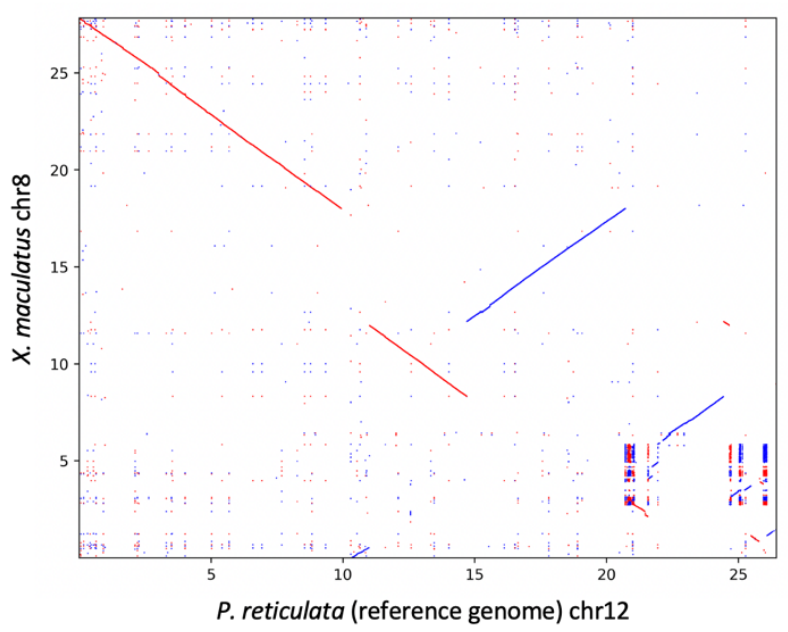

C

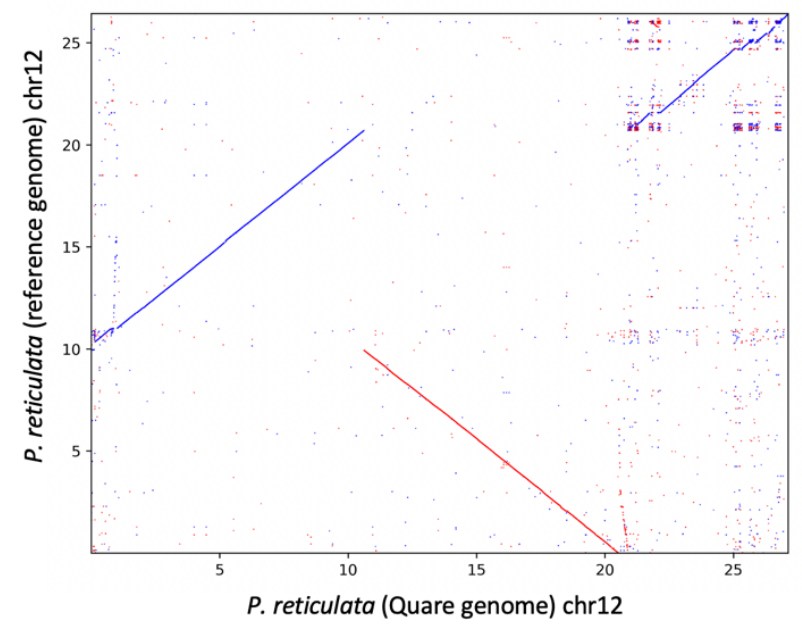

B

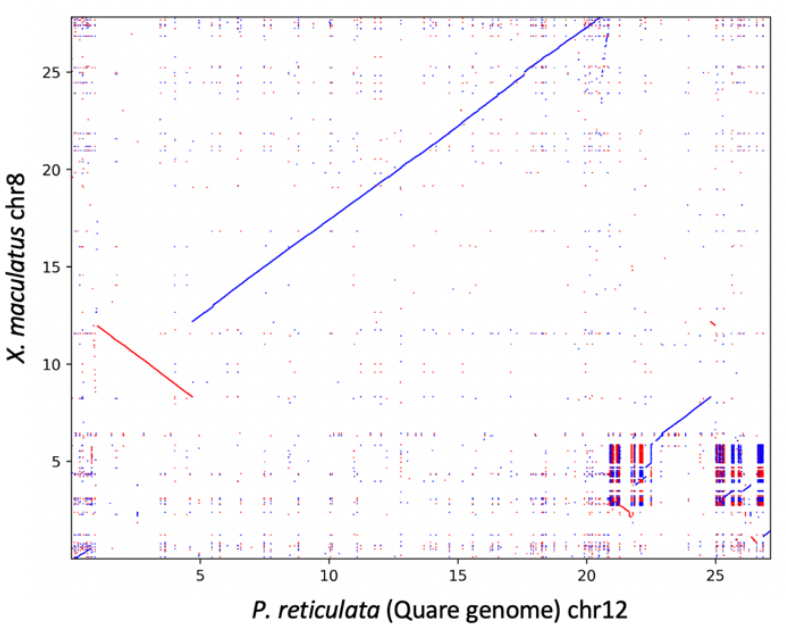

Figure 4. Structural rearrangements and duplications between $P$. reticulata and $X$. maculatus genomes. Dot-plots of alignments between (A) $X$. maculatus chromosome 8 and $P$. reticulata chromosome 12 from the reference genome assembly (Kunstner et al. 2016), (B) X. maculatus chromosome 8 and $P$. reticulata chromosome 12 from the Quare de novo genome assembly (Almeida et al. 2021), and (C) P. reticulata chromosome 12 from the reference genome assembly and chromosome 12 from the Quare de novo genome assembly. Forward alignments are shown in blue and reverse alignments in red. 
Table 2. Percentage of concordant, properly paired* read alignments

\begin{tabular}{llcccc}
\hline Data & $\begin{array}{l}\text { Sequencing data } \\
\text { Genome assembly }\end{array}$ & P. reticulata & X. maculatus & P. wingei & X. maculatus \\
\hline $\begin{array}{l}\text { bwa -aln/-sampe } \\
\text { Wright et al. 2017 }\end{array}$ & 59 & 5 & 75 & 17 \\
$\begin{array}{l}\text { bothod } \\
\text { bowtie2 -local } \\
\text { Kirkpatrick et al. 2020 } \\
\text { bwa -mem -M } \\
\text { Bergero et al. 2019 }\end{array}$ & 83 & 56 & 87 & 71 \\
\hline $\begin{array}{l}\text { *Both mates of a read pair map to the same chromosome or scaffold, with the expected insert size } \\
\text { and read orientation. }\end{array}$
\end{tabular}

Regions of the sex chromosomes where recombination has recently been halted or greatly suppressed still retain a high degree of similarity between $X$ and $Y$ sequences. They are also expected to show an elevated SNP density in males compared to females, as Y-linked reads carrying Y-specific polymorphisms will still align to the homologous $X$ region of the female reference genome (Fig. 1; Vicoso et al. 2013). We observed this pattern in $P$. wingei (Darolti et al. 2019) and in replicate upstream populations of $P$. reticulata (Wright et al. 2017; Almeida et al. 2020), and we designated this as Stratum II. It is important to note that in contrast to Stratum I, Stratum II appears to have formed independently several times. Therefore, to further quantify divergence between the sex chromosomes we investigated SNP density differences between the sexes using several methodological approaches. In P. reticulata, we observe a significantly elevated male SNP density on the sex chromosomes in both of the analyses that aligned reads to the de novo $P$. reticulata genome (Wilcoxon rank sum test $p<0.001$, Fig. 5A, B). By contrast, the SNP density profiles of the autosomes and the sex chromosomes were indistinguishable in all the analyses that used $X$. maculatus as the reference genome (Fig. 5D, E, F), due to the accumulation of numerous fixed differences between $P$. reticulata and $X$. maculatus which conceal the subtle polymorphisms differences between $P$. reticulata males and females. The $P$. wingei $X$ and $Y$ chromosomes have previously been 293 suggested to be more diverged than those of $P$. reticulata, as shown through more pronounced coverage and SNP density differences between the sexes (Darolti et al. 2019) and a greater accumulation of repetitive sequences on the sex chromosomes in P. wingei compared to P. reticulata (Morris et al. 2018; Almeida et al. 2021). Our results here confirm this, as we find a significantly higher male:female SNP 297 density for the sex chromosomes compared to the autosomes across all methodological analyses, as well as when using either one of the $P$. wingei de novo or the $X$. maculatus genomes (Wilcoxon rank sum test $p<0.001$, Fig. 6). 
Method

SNP calling: bow2pro

Filtering: min. coverage $10, \mathrm{MAF}>0.3$

SNP density: per gene

Wright et al. 2017
SNP calling: bcftools

Filtering: QUAL 20, min. read depth 3

SNP density: per $50 \mathrm{~kb}$ windows

Kirkpatrick et al. 2020

SNP calling: GATK

Filtering: QUAL 30, min. read depth 20

SNP density: per $50 \mathrm{~kb}$ windows

Bergero et al. 2019
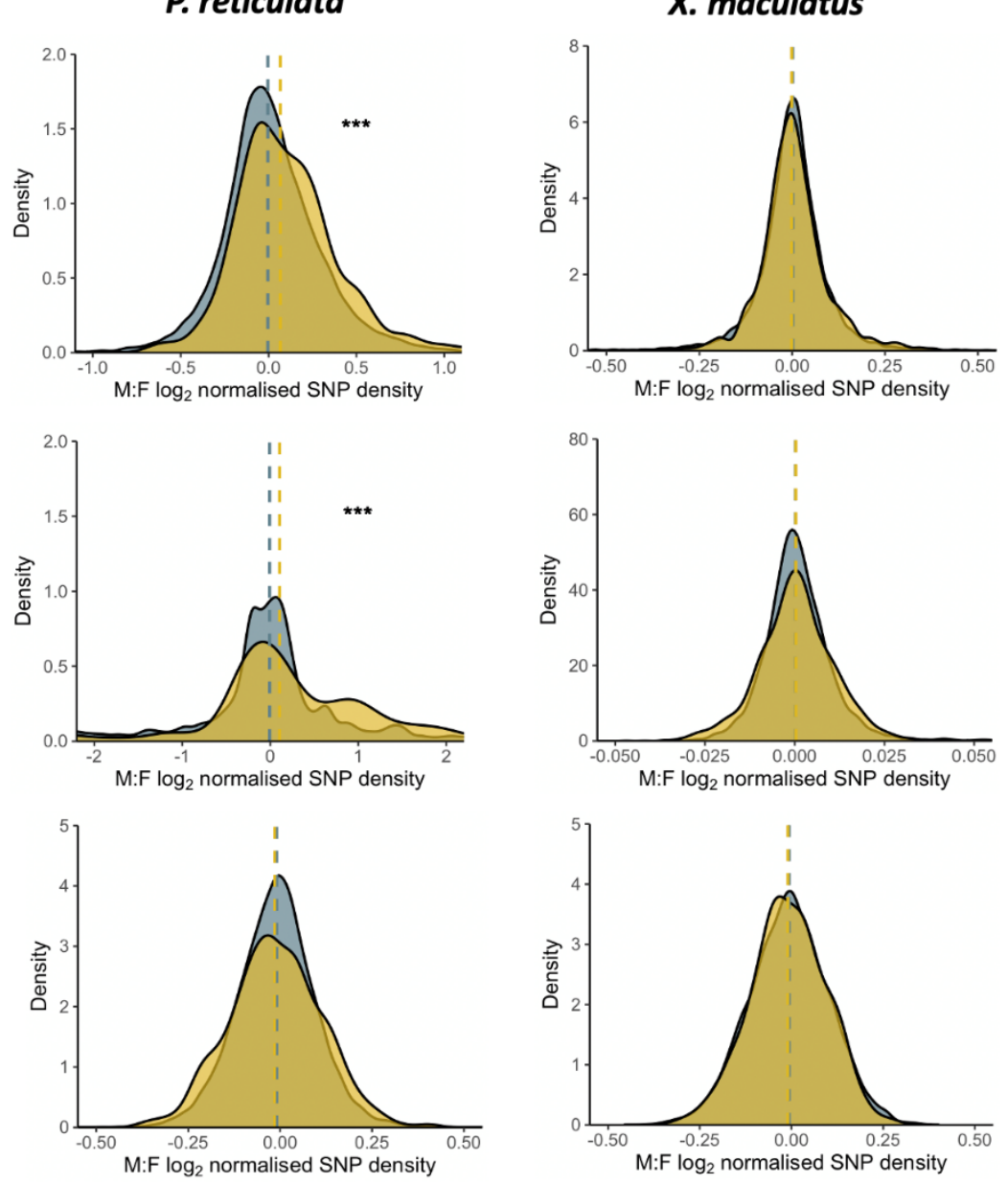

Figure 5. Distribution of $P$. reticulata male:female SNP density for the autosomes (gray) and the sex

chromosomes (yellow). Dashed vertical lines indicate median SNP densities and significant differences between the autosomes and the sex chromosomes are shown $(* * * p$-value $<0.001)$. 
Method

SNP calling: bow2pro

Filtering: min. coverage $10, \mathrm{MAF}>0.3$

SNP density: per gene

Wright et al. 2017
SNP calling: bcftools Filtering: QUAL 20, $\mathrm{min}$. read depth 3 SNP density: per $50 \mathrm{~kb}$ windows Kirkpatrick et al. 2020
SNP calling: GATK

Filtering: QUAL 30, min. read depth 20 SNP density: per $50 \mathrm{~kb}$ windows Bergero et al. 2019
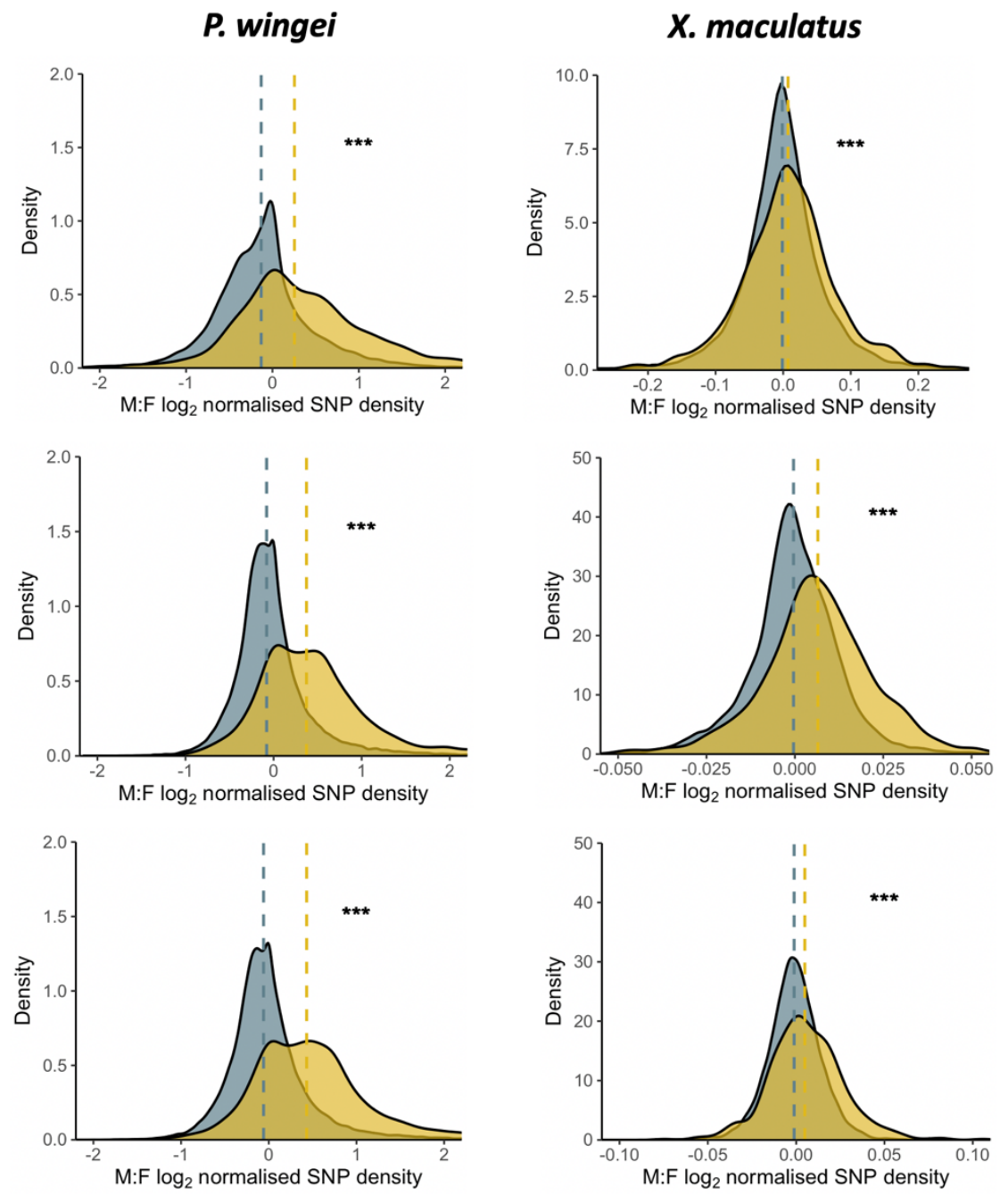

Figure 6. Distribution of $\boldsymbol{P}$. wingei male:female SNP density for the autosomes (gray) and the sex chromosomes (yellow). Dashed vertical lines indicate median SNP densities and significant differences between the autosomes and the sex chromosomes are shown $(* * * p$-value $<0.001)$.

\section{Discussion}

Replication is fundamental to scientific pursuits, and confirmation is necessary to build a robust understanding of the natural world. The expansion of public data efforts has greatly aided transparency and replication efforts, and this remarkable and rapid shift in the scientific culture is exemplified by genomics research, where most of the major journals require deposition of sequencing data as a condition of publication. Failures to replicate results are concerning, and necessitate further work to validate or nullify. However, it is important to understand that different replication approaches will have different risks of Type II errors, or erroneous negative results. This is especially problematic for the detection of subtle, small effect patterns, such as with initial divergence between $\mathrm{X}$ and $\mathrm{Y}$ chromosomes. 
Here we used the same dataset across various methodologies and genome assemblies to test the sensitivity and accuracy of different approaches. Our results show how small changes in the precision of methods can lead to the failure to detect patterns of sex chromosome differentiation in the guppy. The low overall divergence between the $X$ and $Y$ can make detection difficult, but it has nonetheless been observed across multiple datasets, spanning DNA, RNA and methylation data, as well as multiple methods,

323 including comparisons of male and female coverage and SNP density (Wright et al. 2017; Darolti et al. 2019; Almeida et al. 2021), identification of male-specific sequence (Morris et al. 2018; Almeida et al.

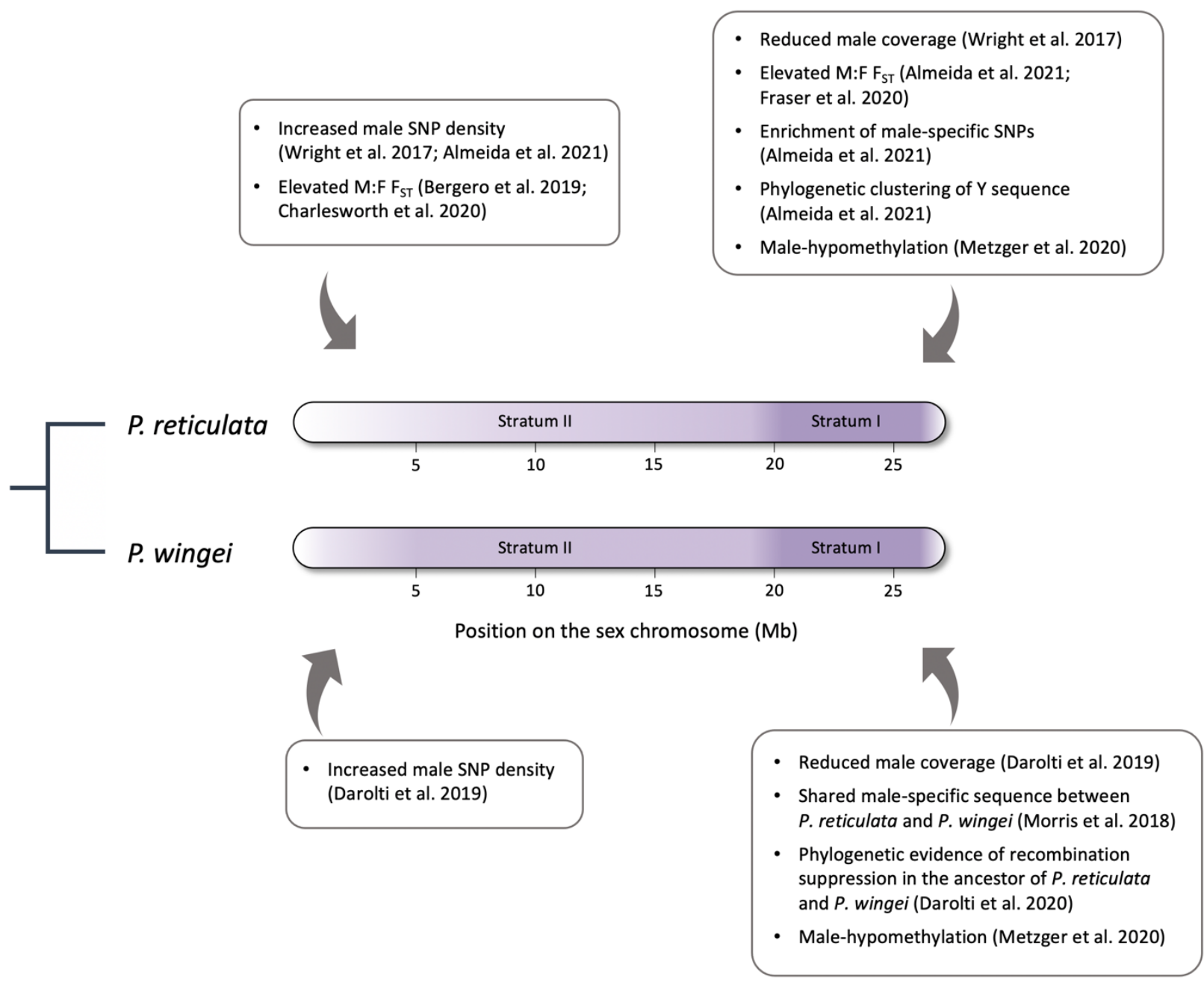

Figure 7. Structure of the $P$. reticulata and $P$. wingei sex chromosomes as predicted by multiple methods, including comparisons of male and female coverage, SNP density, phylogenetic and methylation analyses. 
By using the same sample data across multiple methods and genomes, our results illustrate how important methodological differences can alter perceived results, and highlight the need for replication studies to at minimum replicate the analysis using identical methods on the original or equivalent dataset. When possible, replication efforts should go beyond minimum, and expand the analysis by employing more sophisticated methods on existing or expanded datasets. Despite this, some replication efforts use less sophisticated approaches, and in these cases, there is a real concern that a perceived failure of replication is instead the result of a lack of precision or statistical power. This is particularly problematic in the field of genomics, as there is little consensus about the gold standard in methodologies, particularly with regard to data processing and filtering procedures. The lack of standardized practices, coupled with the rich nature of genomic data and the complexity of genomes can make it difficult to discern subtle but important patterns.

Our approach of evaluating the same underlying data with multiple methods and genomes does not account for natural variation across samples and populations, which is substantial (Wright et al. 2017; Almeida et al. 2021). For our $P$. reticulata samples, we chose individuals from an upstream low predation Quare population which we have previously shown to have an intermediate signal of sex chromosome divergence (Wright et al. 2017; Almeida et al. 2021). Samples from populations with greater or lesser signal, or sampling variation due to differences in inversions, duplications and divergence among

347 individuals may also contribute to observed differences.

\section{Stratum I}

We have previously observed evidence for a small region of ancestral recombination suppression in $P$. wingei and P. reticulata (Wright et al. 2017; Darolti et al. 2019; Almeida et al. 2021). This has been replicated in some studies, for example Fraser et al. (2020) also found evidence of small regions of $Y$

352 divergence, and Kirkpatrick et al. (2020) confirmed the phylogenetic clustering of $Y$ sequence in this 353 stratum. However, other studies (Bergero et al. 2019; Charlesworth et al. 2020; Kirkpatrick et al. 2021) did 354 not fully replicate these findings.

355 It is worth noting that Stratum I region of the guppy $Y$ chromosome is enriched for repetitive elements 356 (Almeida et al. 2021), and reads from this region may, depending on the parameters used, map to 357 repetitive elements across the genome, obscuring real read depth differences between males and females 358 if non-coding sequence is included in the analysis. Focusing on uniquely mapping reads when comparing coverage differences between males and females can minimize issues associated with $\mathrm{Y}$ repetitive regions. 
However, our comparative analysis revealed that a pattern of $X-Y$ differentiation can still be recovered without restricting the analysis to uniquely mapping reads (Fig. 2, Fig. 3). More stringent SNP filtering parameters can also help eliminate noise in genomic comparisons, and this is particularly important when studying young sex chromosomes as they are expected to exhibit subtle divergence signatures. We were, however, able to identify a signal of elevated male SNP density on the sex chromosomes relative to the autosomes, indicative of $Y$ divergence, across several methodological approaches using different degrees of filtering stringency (Fig. 5, Fig. 6).

Beyond mapping parameters, by far the most substantial source of variation in the results of the different pipelines we compared lies in the reference genome used. This is in part due to the extensive structural variation across populations and species (Fig. 4), but also due to sequence evolution. These two factors combined mean that error compounds over phylogenetic distances, and as the distance between the samples and the genome they are mapped to increases, the ability to detect reduced male:female read depth decreases. This is most evidenced in the strategy by Kirkpatrick et al. (2020), who mapped reads from Poecilia species to the Xiphophorus genome. They argued that changes over the 40 my phylogenetic distance separating these genera was outweighed by the fact that the Xiphorphorus genome is more complete. However, the read mapping rate in Table 2 reveals instead that this strategy is less accurate than using less-complete species- or population-specific genome assemblies, as a significantly smaller proportion of Poecilia reads map to the Xiphophorus genome across all methods, thereby reducing usable data. This problem is exacerbated by the substantial structural differences between Xiphophorus and Poecilia on the sex chromosome (Fig. 4), further complicating the comparison. Interestingly, their mapping and filtering methods would have detected Stratum I if they had mapped to a con-specific genome (Fig. 2B, Fig. 3B). To a lesser extent, this is also a problem when mapping data to genomes assembled on different $P$. reticulata populations. The genome used can also greatly affect the perceived patterns of SNP diversity, and relying on the distantly related Xiphophorus genome can obscure a signal of elevated male SNP density on the sex chromosomes due to fixed differences between the target species reads and the Xiphophorus sequence (Fig. 5).

\section{Stratum II}

As recombination is increasingly suppressed in nascent regions of a sex chromosome, we expect the accumulation of Y-specific SNPs, and we observed this in replicate upstream populations of $P$. reticulata (Wright et al 2019; Almeida et al. 2021) and in P. wingei (Darolti et al. 2019), consistent with convergent evolution across populations and species (Darolti et al. 2020). Whether this is due to the important 
environmental effects on recombination rate (Plough 1917; Grell 1971; Stevison et al. 2019), sexual conflict (Wright et al. 2017), neutral shifts in male recombination hotspots (Wright et al 2016; Bergero et al. 2019) or selection against recombinants in the wild remains an important area of further work.

Additionally, given that many mechanisms of recombination suppression only accumulate over time (Furman et al. 2020 and references cited), it also remains unclear how complete recombination suppression is in this region, and whether rare recombination events observed in this region in lab-reared males (Bergero et al. 2019) occur in wild populations. Regardless, it is important to note that suppressed recombination does not necessarily mean that recombination never occurs between the $\mathrm{X}$ and $\mathrm{Y}$ chromosomes, but rather that it is at least exceedingly rare or recombinant individuals are selected against.

Because of the expected heterogeneity observed in the initial stages of the divergence process (Bergero et al. 2013; Natri et al. 2013; Reichwald et al 2015), sliding window approaches may be insufficient to reveal overall patterns of elevated male SNP density expected in these regions. Density distributions or direct statistical comparisons between species may be required. This is evidenced by our observation of elevated male:female SNP density across nearly all methods (Fig. 5 and 6), with the exception of $P$. reticulata data mapped to the Xiphophorus genome, again illustrating the problems with mapping over vast evolutionary distances.

\section{Concluding remarks}

Here we have used the same data to compare methods and genomes in the discovery of nascent sex chromosomes. We hope that our results provide a gold standard for future work in other study systems, and resolve some of the recent controversy over the sex chromosomes in Poecilia.

\section{Acknowledgements}

We thank Jacelyn Shu of jacelyndesigns.com for drafting Fig. 1. We gratefully acknowledge funding from the ERC (grant agreement 680951), NSERC and a Canada 150 Research Chair to JEM.

\section{References}

Almeida, P., Sandkam, B.A., Morris, J., Darolti, I., Breden, F., Mank, J.E. (2021) Divergence and remarkable diversity of the $Y$ chromosome in guppies. Molecular Biology \& Evolution 38, 619-633 
Bergero, R., Qui, S., Forrest, A., Borthwick, H., Charlesworth D. (2013) Expansions of the pseudoautosomal region and ongoing recombination suppression in the Silene latifolia sex chromosomes. Genetics 194, 673-686

Bergero, R., Gardner, J., Bader, B., Yong, L., Charlesworth, D. (2019) Exaggerated heterochiasmy in a fish with sex-linked male coloration polymorphisms. Proceedings of the National Academy of Sciences USA $116,6924-6931$

Bolger, A. M., Lohse, M., \& Usadel, B. (2014). Trimmomatic: a flexible trimmer for Illumina sequence data. Bioinformatics, 30, 2114-2120.

Carvalho, A.B., Clark, A.G. (2013). Efficient identification of $Y$ chromosome sequences in the human and Drosophila genomes. Genome Research 23, 1894-1907

Charlesworth, D., Bergero, R., Graham, C., Gardner, J., Yong, L. 2020. Locating the sex determining region of linkage group 12 of guppy (Poecilia reticulata). G3 10, 3639-3649

Darolti, I., Wright, A.E., Mank, J.E. (2020) Guppy Y chromosome integrity maintained by incomplete recombination suppression. Genome Biology \& Evolution 12, 965-977

Darolti, I., Wright, A.E., Sandkam, B.A., Morris, J., Bloch, N.I., Farré, M., Fuller, R.C., Bourne, G.R. Larkin, D.M., Breden, F., Mank, J.E. (2019) Extreme heterogeneity in sex chromosome differentation and dosage compensation in livebearers. Proceedings of the National Academy of Sciences, USA 116, 19031-19036

Danecek, P., Auton, A., Abecasis, G., Albers, C. A., Banks, E., DePristo, M. A., Handsaker, R. E., Lunter, G., Marth, G. T., Sherry, S. T., McVean, G., Durbin, R., 1000 Genomes Project Analysis Group. (2011). The variant call format and VCFtools. Bioinformatics 27, 2156-2158

Fraser, B.A., Whiting, J.R., Paris, J.R., Weadick, C.J., Parsons, P.J., Charlesworth, D., Bergero, R., Bemm, F., Hoffmann, M., Kottler, V.A., Liu, C., Dreyer, C., Weigel, D. (2020) Improved reference genome uncovers novel sex-linked regions in the guppy (Poecilia reticulata). Genome Biology \& Evolution 12, 1789-1805

Furman, B.L.S., Metzger, D.C.H., Darolti, I., Wright, A.E., Sandkam, B.A., Almeida, P., Shu, J.J., Mank, J.E. (2020) Sex chromosome evolution: So many exceptions to the rules. Genome Biology \& Evolution 12, $750-763$

Grell, R.F., 1971. Heat induced exchange in fourth chromosome of diploid females of Drosophila melanogaster. Genetics 69, 523-527

Kiełbasa, S. M., Wan, R., Sato, K., Horton, P., \& Frith, M. C. (2011). Adaptive seeds tame genomic sequence comparison. Genome research 21, 487-493

Kirkpatrick, M., Dixon, G., Sardell, J.M., Schartl, M., Peichel, C.L. (2020). Evolution of the canonical sex chromosomes of the guppy and relatives. Biorxiv doi: https://doi.org/10.1101/2020.09.25.314112

Kumar, S., Stecher, G., Suleski, M., Hedges, S.B. (2017) TimeTree: A Resource for Timelines, Timetrees, and Divergence Times. Molecular Biology and Evolution 34, 1812-1819

Kunstner, A., Hoffmann, M., Fraser, B.A., Kottler, V.A., Sharma, E., Weigel, D., Dreyer, C. (2016) The genome of the Trinidadian guppy, Poecilia reticulata, and variation in the Guanapo population. PLOS ONE 11, e0169087

Langmead, B., Salzberg, S. L. (2012). Fast gapped-read alignment with Bowtie 2. Nature methods 9, 357359 
Li, H. (2011). A statistical framework for SNP calling, mutation discovery, association mapping and population genetical parameter estimation from sequencing data. Bioinformatics, 27, 2987-2993

Li, H., Durbin, R. (2009) Fast and accurate short read alignment with Burrows-Wheeler transform. Bioinformatics 25, 1754-1760

Li, H., Handsaker, B., Wysoker, A., Fennell, T., Ruan, J., Homer, N., Marth, G., Abecasis, G., Durbin, R., 1000 Genome Project Data Processing Subgroup (2009). The sequence alignment/map format and SAMtools. Bioinformatics 25, 2078-2079

McKenna, A., Hanna, M., Banks, E., Sivachenko, A., Cibulskis, K., Kernytsky, A., Garimella, K., Altshuler, D., Gabriel, S., Daly, M., DePristo, M. A. (2010). The Genome Analysis Toolkit: a MapReduce framework for analyzing next-generation DNA sequencing data. Genome research 20, 1297-1303

Metzger, D.C.H., Mank, J.E. (2020) Conserved sex-biased DNA methylation patterns target key developmental genes and non-recombining region of the guppy sex chromosome. Biorxiv doi: https://doi.org/10.1101/2020.08.21.261792

Morris, J., Darolti, I., Bloch, N.I., Wright, A.E., Mank, J.E. (2018). Shared and species-specific patterns of nascent $Y$ chromosome evolution in two guppy species. Genes 9,238

Nanda, I., Schartl, M., Epplen, J.T., Feichtinger, W., Schmid, M (1993) Primitive sex chromosomes in poecilid fishes harbour simple repetitive DNA sequences. Journal of Experimental Biology 265, 301308

Natri, H.M., Shikano, T., Merila, J. (2013) Progressive recombination suppression and differentiation in recently evolved neo-sex chromosomes. Molecular Biology \& Evolution 30, 11311144

Palmer, D.H., Rogers, T.F., Dean, R., Wright, A.E. (2019) How to identify sex chromosomes and their turnover. Molecular Ecology. 28, 4709- 4724

Plough, H.H. 1917. The effect of temperature on crossing over in Drosophila. Journal of Experimental Zoology 24, 147-209

Postlethwait, J.H., Warren, W.C. (2013) The genome of the platyfish, Xiphophorus maculatus, provides insights into evolutionary adaptation and several complex traits. Nature Genetics 45, 567-572

Quinlan, A. R., \& Hall, I. M. (2010). BEDTools: a flexible suite of utilities for comparing genomic features. Bioinformatics 26, 841-842

R Core Team (2019) R: A language and environment for statistical computing. R Foundation for Statistical Computing, Vienna.

Reichwald, K., et al. (2015) Insights into sex chromosome evolution and agine from the genome of a short-lived fish. Cell 163, 1527-1538

Sandkam, B.A., Almeida, P., Darolti, I., Furman, B.L.S., van der Bijl W., Morris, J., Bourne, G.R., Breden, F., Mank, J.E. (2021) Extreme $Y$ chromosome polymorphism corresponds to five male reproductive morphs in a freshwater fish. Nature Ecology \& Evolution 5, 939-948

Schartl, M., Walter, R.B., Shen, Y.J., Garcia, T., Catchen, J., Amores, A., Braasch, I., Chalopin, D., Volff, J.N., Lesch, K.P., Bisazza, A., Minx, P., Hillier, L., Wilson, R.K., Fuerstenberg, S., Boore, J., Searle, S., 
Postlethwait, J.H., Warren, W.C. (2013). The genome of the platyfish, Xiphophorus maculatus, provides insights into evolutionary adaptation and several complex traits. Nature genetics 45, 567-572

500 Smeds, L., Warmuth, V., Bolivar, P., Uebbing, S., Burri, R., Suh, A., Ellegren, H. (2015). Evolutionary

501 analysis of the female-specific avian W chromosome. Nature Communications 6,7330

502 Stevison, L.S., Sefich, S., Chase, R., Graze, R.M. (2017). Recombination rate plasticity: revealing

503 mechanisms by design. Philosophical Transactions of the Linnean Society, B. 372, 20160459

504 Vicoso, B., Bachtrog, D. (2015). Numerous transitions of sex chromosomes in Diptera. PLOS Biology, 13, 505 e1002078

506 Vicoso, B., Bachtrog, D. (2013). Reversal of an ancient sex chromosome to an autosome in Drosophila.

507 Nature, 499, 332-335

508 Vicoso, B., Emerson, J. J., Zektser, Y., Mahajan, S., \& Bachtrog, D. (2013). Comparative sex chromosome 509 genomics in snakes: differentiation, evolutionary strata, and lack of global dosage compensation. PLoS 510 biology, 11, e1001643

511 Wright, A.E., Dean, R., Zimmer, F., Mank, J.E. (2016) How to make a sex chromosome. Nature 512 Communications 7, 12087

513 Wright, A.E., Darolti, I., Bloch, N.I., Oostra, V., Sandkam, B., Buechel, S.D., Kolm, N., Breden, F., Vicoso, 514 B., Mank, J.E. (2017) Convergent recombination suppression suggests a role of sexual selection in guppy 515 sex chromosome formation. Nature Communications 8: 14251 